\section{La obesidad y sus tendencias en la Región}

\author{
Manuel Peña ${ }^{1}$ \\ y Jorge Bacallao ${ }^{2}$
}

En la Región de las Américas ha habido, en las últimas décadas, un notable incremento de las enfermedades crónicas no trasmisibles (ECNT) asociadas a estilos de vida no saludables. Estas enfermedades y trastornos en conjunto, entre los que se encuentran la hipertensión arterial, la diabetes mellitus tipo 2, la enfermedad coronaria, los accidentes cerebrovasculares, la obesidad y algunos tipos de cáncer, son las principales causas de muerte, morbilidad, discapacidad y deterioro de la calidad de vida.

Otro aspecto crítico de esta epidemia de ECNT es el alto costo que representan para los servicios de salud el tratamiento y la rehabilitación de pacientes con estas afecciones, factores que acentúan las inequidades existentes.

Entre las ECNT, la obesidad merece especial atención, ya que es en sí misma una enfermedad crónica y a la vez un reconocido factor de riesgo de muchas otras. El estudio y abordaje de la obesidad no pueden desligarse de los de otras ECNT por diversas razones: 1) porque comparten algunos factores causales y subyacentes comunes, como la alimentación inapropiada y el sedentarismo; 2) porque al identificar a los sujetos obesos se está identificando a una alta proporción de los sujetos en riesgo de padecer otras ECNT; 3) porque al prevenir la obesidad -mediante la promoción de estilos de vida saludables - se previenen la mayoría de las ECNT; $\mathrm{y}$, por último, 4) porque al tratar al obeso se disminuye el riesgo de que sufra complicaciones y también se reduce el efecto mediador que tiene la obesidad en relación con otros factores de riesgo.

El cuadro 1 muestra la prevalencia del exceso de peso (25 - 29,9 $\mathrm{kg} / \mathrm{m}^{2}$ ) y de la obesidad (30 o más $\mathrm{kg} / \mathrm{m}^{2}$ ) en mujeres de algunos países de América Latina y del Caribe (1). Los valores más elevados corresponden a Jamaica, país que podría ser representativo de lo que ocurre en la mayoría de los países anglófonos del Caribe. Puede apreciarse que, con la excepción de Haití, más de $23 \%$ de la población de mujeres entre las edades de 15 y 49 años están en sobrepeso y más de un tercio presenta sobrepeso u obesidad. Es de señalar que estos datos, que corresponden a los de la mayoría de los países pobres, son reveladores de la magnitud de las tendencias en la Región.

Según algunos estudios diacrónicos, los cambios se producen claramente en el sentido del aumento. En un estudio realizado en Estados Unidos (2) se notificó que en la población de origen hispano se ha incrementado la proporción de obesos de $11,6 \%$ en 1991 a 20,8\% en 1998 (80\% de incremento), mucho más que en otros grupos étnicos.

Brasil, que dispone de datos correspondientes a tres encuestas realizadas en 1975, 1989 y 1996, muestra un aumento superior al 100\% en la proporción de mujeres obesas de 20 a 34 años de edad (3). Otros datos, disponibles solo para 1975 y 1989, muestran también un marcado incremento de la obesidad en todos los estratos de población encuestados (4).

El Ministerio de Salud Pública de Costa Rica, basado en la Encuesta Nacional de Nutrición de 1996, notificó un aumento de 34,6 a 45,9\% de las mujeres con sobrepeso y obesas entre los 20 y 44 años de edad de 1982 a 1996. En el grupo de mujeres de 45 a 59 años el aumento fue de 56 a $75 \%$ (5). 
CUADRO 1. Prevalencia de exceso de peso y de obesidad en mujeres de 15 a 49 años de edad en países de América Latina y el Caribe

\begin{tabular}{lrccc}
\hline \multicolumn{1}{c}{ País/año } & $n$ & IMCa/DE & $\begin{array}{c}\text { Exceso de peso } \\
\left(25<x<30 \mathrm{~kg} / \mathrm{m}^{2}\right) \\
\%\end{array}$ & $\begin{array}{c}\text { Obesidad } \\
\left(\geq 30 \mathrm{~kg} / \mathrm{m}^{2}\right) \\
\%\end{array}$ \\
\hline Bolivia/1994 & 2,347 & $24,3 / 3,7$ & 26,2 & 7,6 \\
Brasil/1996 & 3,158 & $24,0 / 4,3$ & 25,0 & 9,7 \\
Colombia/1995 & 3,319 & $24,5 / 4,0$ & 31,4 & 9,2 \\
Guatemala/1995 & 4,978 & $24,2 / 3,9$ & 26,2 & 8,0 \\
Haití/1994-1995 & 1,896 & $21,2 / 3,4$ & 8,9 & 2,6 \\
Honduras/1996 & 885 & $23,5 / 4,7$ & 23,8 & 7,8 \\
Jamaica/1998 & 442 & - & 33,2 & 29,3 \\
México/1987 & 3,681 & $23,7 / 4,3$ & 23,1 & 10,4 \\
Perú/1996 & 10,747 & $25,1 / 3,6$ & 35,5 & 9,4 \\
República & & & & \\
$\quad$ Dominicana/1996 & 7,356 & $24,3 / 4,9$ & 26,0 & 12,1 \\
\hline Fuente: Martorell R, et al. $(6)$. & & & &
\end{tabular}

Fuente: Martorell R, et al. (6).

a IMC = índice de masa corporal.

b $D E$ = desviación estándar.

c Wilks R (datos inéditos).

En la República Dominicana, a partir de las encuestas correspondientes a los Demographic Health Surveys, el incremento de sobrepeso fue de $18,6 \%$ en 1981 a $26 \%$ en 1996 en mujeres de 14 a 49 años de edad, y el aumento de la obesidad fue de 7,3 a $12,1 \%$ (6).

Chile ha notificado incrementos notables de la obesidad en la población de 0 a 6 años entre 1985 y 1995, usando como criterio más de 2 desviaciones estándar (DE) en relación con el patrón de NCHS/CDC (7). En Cuba, los datos disponibles de 1972 a 1982 mostraron incrementos significativos de un exceso de peso moderado ( $>2 \mathrm{DE}$ ) y grave $(>3 \mathrm{DE})(8)$. En 1993 hubo un descenso de las cifras de sobrepeso y obesidad (9) y, aunque no ha sido documentado, en los últimos años se ha notado, sobre todo en la población adulta, un nuevo incremento en la prevalencia de obesos que se relaciona con la recuperación económica y la consecuente mayor disponibilidad de alimentos en el país.

A pesar de limitaciones de diversa índole, tales como la falta de estudios longitudinales, dificultades de muestreo y problemas metodológicos en la recolección de datos antropométricos, no cabe duda de que, según indican los datos de diversos países, la obesidad va en franco ascenso, predomina en las zonas urbanas y es más frecuente en la población femenina, adulta y pobre (1).

\section{¿Qué factores subyacentes explican esta epidemia?}

La obesidad, es por definición, un exceso de grasa corporal que se genera cuando el ingreso energético - el alimentario- es superior al gasto energético — determinado principalmente por la actividad física-durante un período suficientemente largo. Este desequilibrio se ve influenciado por la compleja interacción de factores genéticos, conductuales y del ambiente físico y social.

Aunque en años recientes se han identificado factores genéticos que explican la mayor susceptibilidad de algunos individuos a la obesidad, el incremento tan abrupto de la obesidad que ha ocurrido en las últimas 
décadas y su gran extensión obedecen principalmente a cambios importantes en la alimentación de la población (10), al patrón de actividad física (1) y a otros factores de índole sociocultural.

1. Cambios más relevantes en los patrones alimentarios: Mayor consumo de alimentos de alta densidad energética y bajo contenido de nutrientes.

Alto consumo de azúcares y carbohidratos refinados.

Alto consumo de grasas saturadas, ácidos grasos monoinsaturados "trans" y colesterol.

Alto consumo de bebidas alcohólicas.

Pobre consumo de vegetales y frutas frescas.

Aumento del tamaño de las raciones de alimentos, especialmente en restaurantes y "cadenas de alimentos rápidos".

2. Reducción de la actividad física:

Reducción del trabajo físico debido a adelantos tecnológicos.

Uso cada vez mayor de transporte automotor.

Automatización de los vehículos y reducción del gasto energético en la operación de maquinarias y vehículos (cierre y ventanillas electrónicas, p. ej.)

Uso de ascensores y "escaleras rodantes".

Reducción del tiempo dedicado a jugar al aire libre por inseguridad y preferencia de los juegos electrónicos y la televisión.

3. Aspectos socioculturales:

Concepto de la obesidad como expresión de salud y no de enfermedad.

Concepto de la obesidad como sinónimo de afluencia en algunas culturas.

Aceptación de la obesidad debido al patrón cultural de belleza por influencia de los medios de comunicación.

Para controlar la epidemia de obesidad y de otras ECNT que afectan a todos los estratos sociales de la población, así como a la mayoría de los países de nuestra Región, la acción clave es prevenir. La prevención no puede depender exclusivamente de pautas dictadas al individuo - como ocurre con frecuencia, - sino en crear las oportunidades para que los estilos de vida saludables estén al alcance de toda la población. De ahí que la promoción de la salud sea la estrategia de lucha fundamental, procurando abarcar no solo al individuo, sino a todos los componentes de la sociedad; actuar en los espacios donde transcurre la vida individual y social; promulgar leyes, y elaborar políticas encaminadas a mejorar el entorno físico y social.

\section{REFERENCIAS}

1. Peña M, Bacallao J. La Obesidad en la pobreza: un problema emergente en las Américas. En: Peña M, Bacallao J, eds. La obesidad en la pobreza: un nuevo reto para la salud pública. Washington, D. C.: Organización Panamericana de la Salud; 2000. pp. 3-11. (Publicación Científica No. 576).

2. Mokdad AH, Serdula MK, Dietz WH, Bowman BA, Marks JS, Koplan JP, et al. The spread of the obesity epidemic in the United States, 1991-1998. JAMA. 1999;282:15191522. 
3. Monteiro CA, D'A Benicio MH, Conde WL, Popkin BM, et al. Shifting obesity trends in Brazil. Eur J Clin Nutr 2000;54:342-346.

4. Sichieri R, Coitinho DC, Leao MM, Recine E, Everhart JE, et al. High temporal, geographic, and income variation in body mass index among adults in Brazil. Am J Public Health 1994;84:793-798.

5. Costa Rica, Ministerio de Salud. Encuesta Nacional de Nutrición. San José, Costa Rica: Fascículo de antropometría; 1996.

6. Martorell G, Khan LK, Hughes ML, Grummer-Strawn LM, et al. Obesity in Latin American women and children. J Nutr 1998;128:1464-1473.

7. Albala C, Vio F. Obesidad y pobreza: un desafío pendiente en Chile. En: Peña M, Bacallao J, eds. La obesidad en la pobreza: un nuevo reto para la salud pública. Washington, D.C.: Organización Panamericana de la Salud; 2000. pp. 46-56. (Publicación científica No. 576).

8. Esquivel M, Romero JM, Berdasco A, Gutierrez JA, Jimenez JM, Posada E, et al. Estado nutricional de preescolares de Ciudad de La Habana entre 1972 y 1993. Rev Panam Salud Publica 1997;1:349-354.

9. Porrata C, Rodríguez-Ojea A, Jiménez S. La transición epidemiológica en Cuba. En: Peña M, Bacallao J, eds. La obesidad en la pobreza: un nuevo reto para la salud pública. Washington, D.C.: Organización Panamericana de la Salud; 2000. pp. 57-72. (Publicación Científica No. 576).

10. Popkin BM. The nutrition transition and its implications in lower-income countries. Public Health Nutr 1998;1:5-21.

11. Popkin BM, Doak C. The obesity epidemic is a worldwide phenomenon. Nutr Rev 1998;56:106-114. 